\title{
SOME RESULTS ON SOLAR ACTIVITY AT 408 MHz
}

\author{
B. Clavelier \\ (Observatoire de Meudon (S. et O.), France)
}

\begin{abstract}
A BST R ACT
We present the first results obtained with the $408 \mathrm{MHz}$ interferometer of Nançay. The typical activity observed at $408 \mathrm{MHz}$ is the 'noise storm'. Undoubtedly with this apparatus we could distinguish multiple centres, generally double.

The comparison with corresponding optical observations and with Nançay observations at frequency $169 \mathrm{MHz}$ allowed us to associate the centres of noise storm at $408 \mathrm{MHz}$ with eruptive centres lying in 'Anomalous Active Regions' and to describe the schematical structure of active zones.
\end{abstract}

The results presented here have been obtained with the $408 \mathrm{MHz}$ interferometer that we have built at Nançay (Clavelier, 1967).

The $1.5 \mathrm{~km}$ base line gives 1.7 resolving power with an interlobe of $25^{\prime}$. This interferometer allows us to measure the East-West dimension of solar sources greater

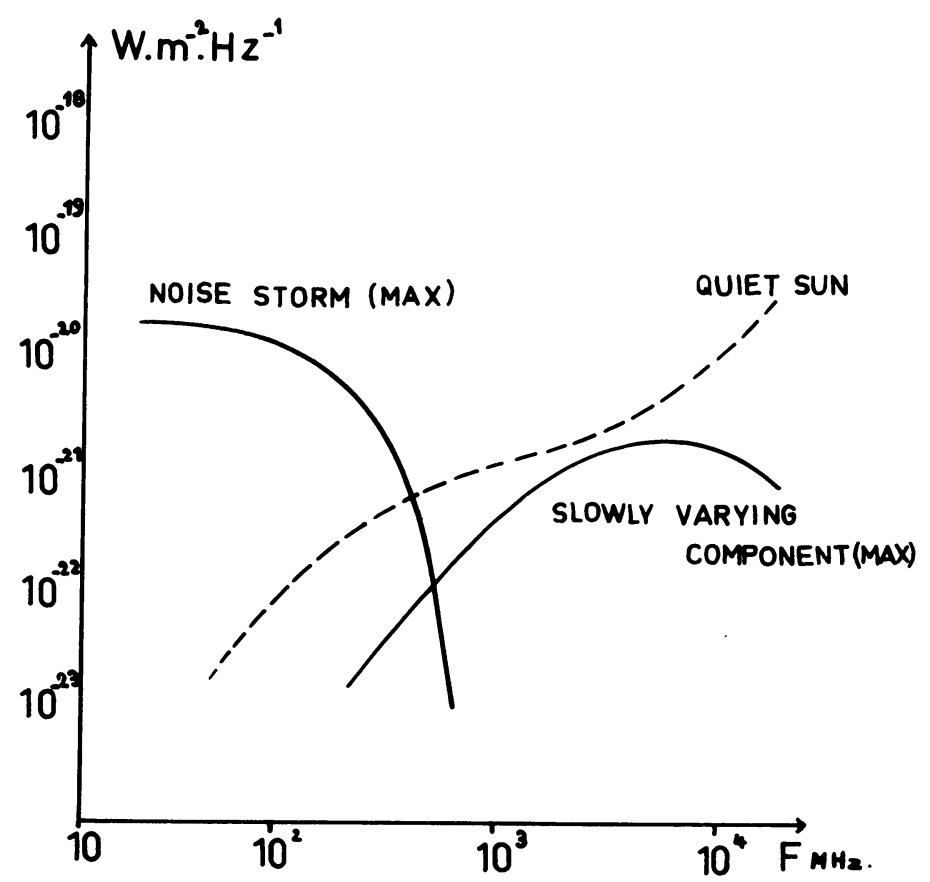

FIG. 1. Spectra of noise storms and slowly varying component. 
than $1^{\prime}$ of arc, and to separate two sources 1.7 distant. The position of an emitting centre on the solar disk is determined with an accuracy of $20^{\prime \prime}$ (of arc).

With this instrument we can analyse centres with a flux greater than $10^{-23} \mathrm{~W} \cdot \mathrm{m}^{-2}$. $\mathrm{Hz}^{-1}$.

The following results are derived from observations carried out during the period from October 1, 1965 to July 14, 1966.

The spectrum of the slowly varying component shows us that the fluxes at $408 \mathrm{MHz}$ are weaker than those at higher frequencies. Likewise, fluxes from noise storms must be weaker, at $408 \mathrm{MHz}$ than for metric wavelengths (Figure 1).

Our observations confirm the weakness of the mean flux (Figure 2): $2 \times 10^{-22}$ $\mathrm{W} \cdot \mathrm{m}^{-2} \cdot \mathrm{Hz}^{-1}$ for $90 \%$ of the centres.

On the other hand, whereas one thought of meeting mainly the slowly varying component at $408 \mathrm{MHz}$, we found that the noise storm activity was the most important. Finally, besides the bursts that we will not discuss here, the two most durable types of emission are given in Table 1.

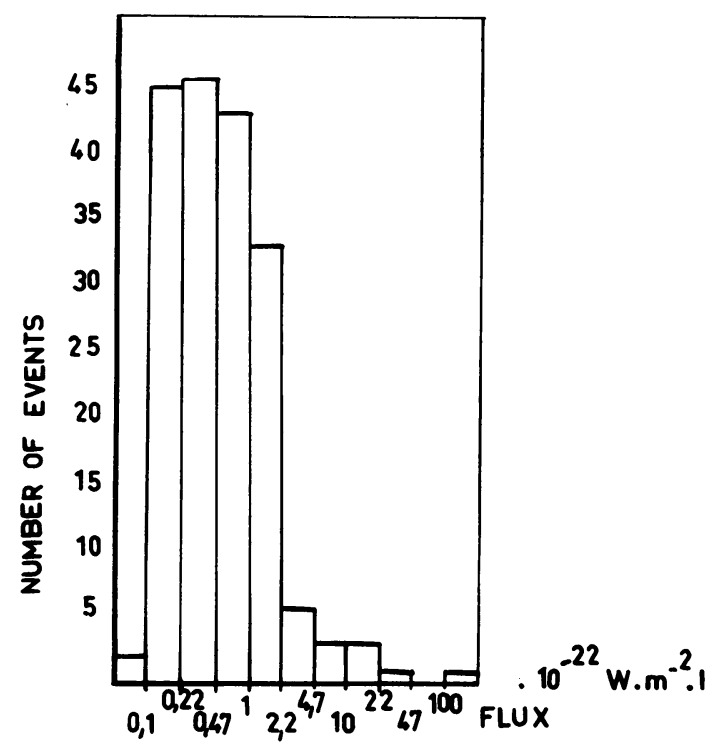

FIG. 2. Histogram of flux densities.

Table 1

Slowly Varying Component

Large $>2: 5$

Brightness temperature $T_{\mathrm{b}}<5 \times 10^{6}{ }^{\circ} \mathrm{K}$

No storms at $169 \mathrm{MHz}$

Not variable

Associated with faculae
Noise storms

Narrow $<2^{\prime}$

$T_{\mathrm{b}}>5 \times 10^{6}{ }^{\circ} \mathrm{K}$

Storm simultaneously at $169 \mathrm{MHz}$

Variable (+ some types 1)

Associated with active centres (with spots) 

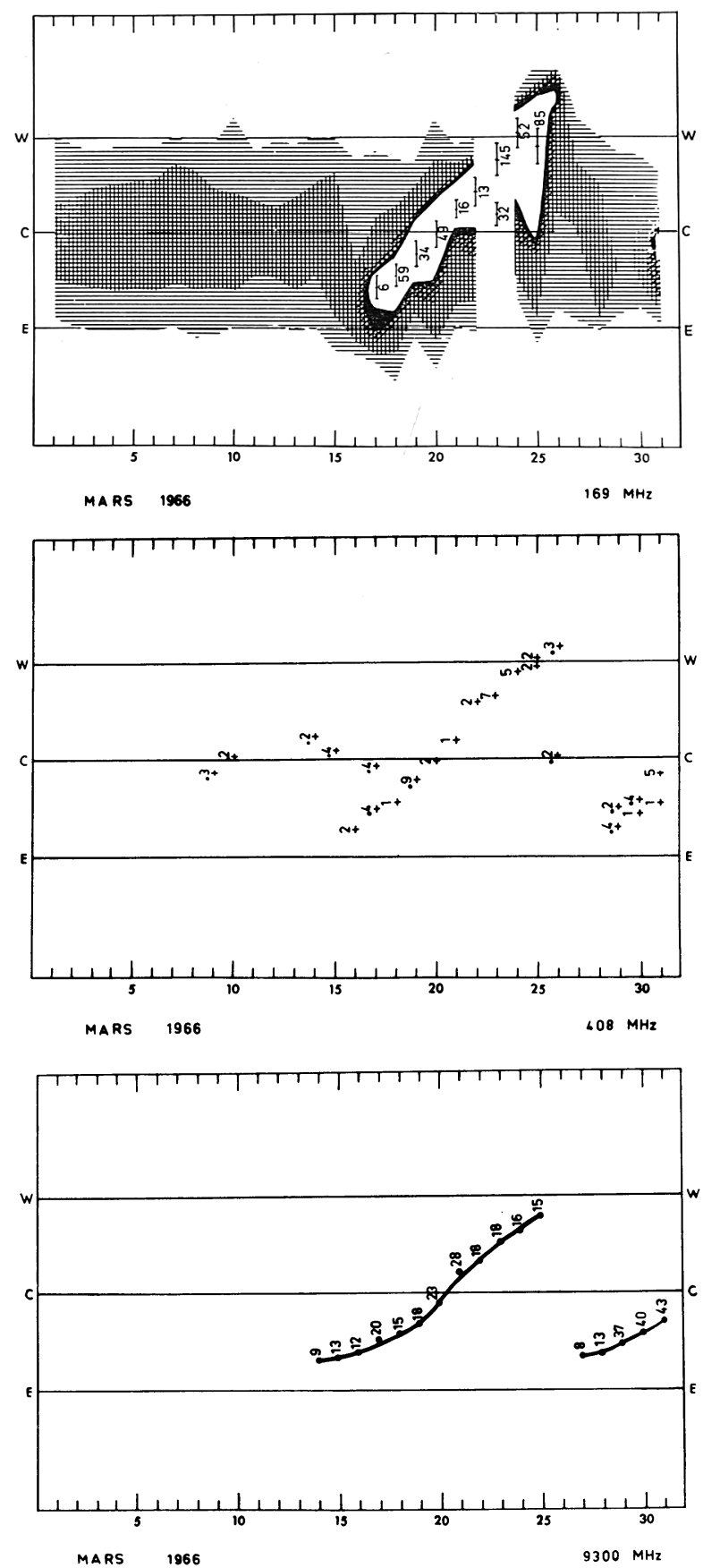

FIG. 3. Maps of solar activity at 9300, 408 and $169 \mathrm{MHz}$ (March 1966). 
In order to study certain properties of the radio centres, we have been led to associate the radioelectric centres with optical centres. For that purpose, we made use of the solar photographs of the Meudon-Observatory and the lists of centres found in the Quarterly Bulletin on Solar Activity.

Among 175 centres observed at $408 \mathrm{MHz}, 123$ were associated with centres of activity where spots were visible, 46 were associated with diffuse faculae without spot; they are coronal condensations of low brightness temperature and of large diameter, and 6 were ambiguous.

With the three interferometers (at $169 \mathrm{MHz}, 408 \mathrm{MHz}$, and $9300 \mathrm{MHz}$ ) of the radio observatory at Nançay we establish a monthly map of solar radioelectric activity giving position and flux of the radio centres. These maps (Figure 3 ) allow us to follow the evolution of solar activity at $408 \mathrm{MHz}$ and to compare it with corre-

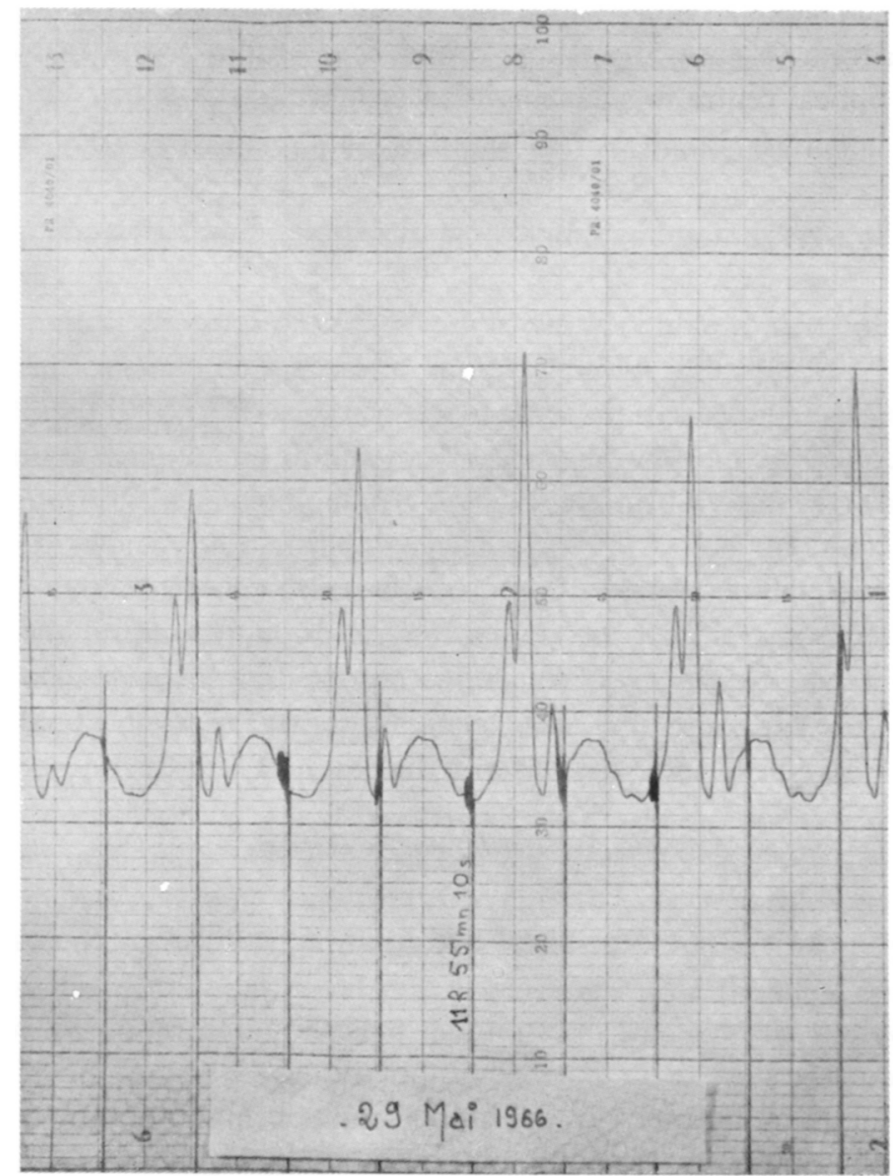

FIG. 4. Record of May 29, 1966, showing a double centre. 
sponding activities at 169 and $9300 \mathrm{MHz}$. From these maps we conclude that the centres at $408 \mathrm{MHz}$ are more stable than those at $169 \mathrm{MHz}$ but less stable than centres at 9300 .

On certain days we can observe two centres close enough to be associated with the same active centre. We are dealing here with multiple centres (generally double) as confirmed by the associated optical centres (Figure 4).

This had not yet been firmly established for this type of activity (Le Squeren had thought of associating two centres at $169 \mathrm{MHz}$, with the same active region in only a few cases (Le Squeren, 1963)). For all these double centres at $408 \mathrm{MHz}$ the corresponding optical centres appear as two distinct regions with spots. We shall see that these two regions correspond to two different eruptive zones each associated with a radio component.

Two methods for measuring altitude give an average height between 70000 and $80000 \mathrm{~km}$.

On the diagram (Figure 5) we note $d$ and $\delta: d=$ radio centre to solar meridian distance, $\delta=$ optical centre to solar meridian distance.

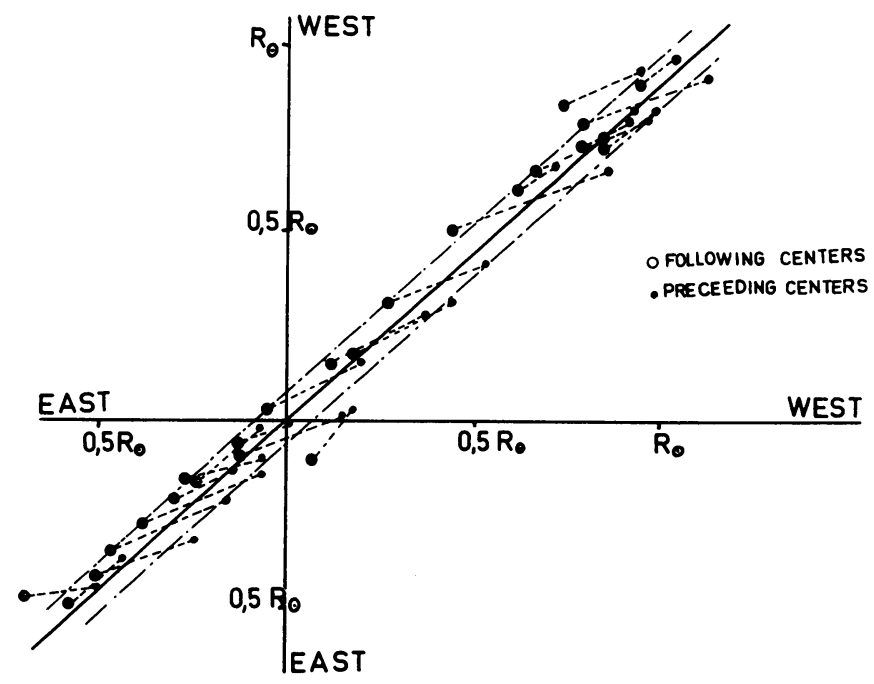

FIG. 5. Height (double centres).

Preceding centres are noted by a dot and following centres by a cross. We found that:

(1) The average slope of the lines joining preceding and following centres shows that the radio centres are more distant (on the average) than the optical centres by a factor of 2 (the mean distance between radio centres being $3^{\prime}$ of arc).

(2) Preceding centres are found on a parallel to $D(70000 \mathrm{~km}$ of altitude $)$ shifted to the West and following centres to the East (average altitude: $70000 \mathrm{~km}$ ). 
Let us explain now, how we have associated the noise storms centres at $408 \mathrm{MHz}$ with the eruptive activity. Although the activity at $408 \mathrm{MHz}$ notably differs from that observed at lower frequencies, we may think that it shows a certain analogy.

In particular, former studies have shown (Le Squeren, 1963) that the centres of activity in the metric range appear following flares.

In the following we will use a very simplified classification of the active regions derived from Martres et al. (1966):

(1) The simple ARs with lines of polarity inversion slightly inclined on the meridian.

(2) The complex ARs for which the opposite polarities are irregularly located with respect to several lines of inversion. In the simple Active Regions they distinguish between:

(a) The simple and normal ARs that present no anomaly as to magnetic structure and aspect of the group of spots, and

(b) The simple but anomalous ARs having either an anomalous inclination of the group of spots on the parallels or a following spot greater than the preceding spot.

Thereafter we will only retain the following classes:

(1) Normal ARs corresponding to simple and normal ARs defined above (not eruptive).

(2) Anomalous ARs corresponding to simple and anomalous ARs and to complex ARs (eruptive).

From the optical observations of 'Meudon' we were able to determine which class of AR our centres could be associated with.

We have found that, with the exception of three centres belonging to a normal AR, all other centres (simple and double) are associated with an anomalous AR.

Inversely most of the anomalous AR are accompanied by $408 \mathrm{MHz}$ storm activity. It is of interest to mention that the two events of July 5, 1966 and September 3, 1966 are two important exceptions: these cases show an obvious anomalous configuration with numerous eruptions; however, no activity at $408 \mathrm{MHz}$ was detected. Those events were accompanied with proton emissions.

It is possible to describe further the association between radio centre and AR, by studying the eruptive zones that appeared in these regions during the days of observations.

In the case of double radio-electric centres, despite the great distance between chromospheric AR and radio centres $(70000 \mathrm{~km})$ we have tried to associate them with two distinct eruptive centres in the same active region. For that purpose we have considered as distinct two groups of eruptive centres more than 1'.7 apart (resolving power of our instrument). We found that out of 17 cases of double radio centres, 10 correspond to two eruptive zones located in the same AR and more than 1'.7 apart.

The conclusion of this comparative study with optical observations is that the noise-storm type activity at $408 \mathrm{MHz}$ can, beyond doubt, be associated with the 


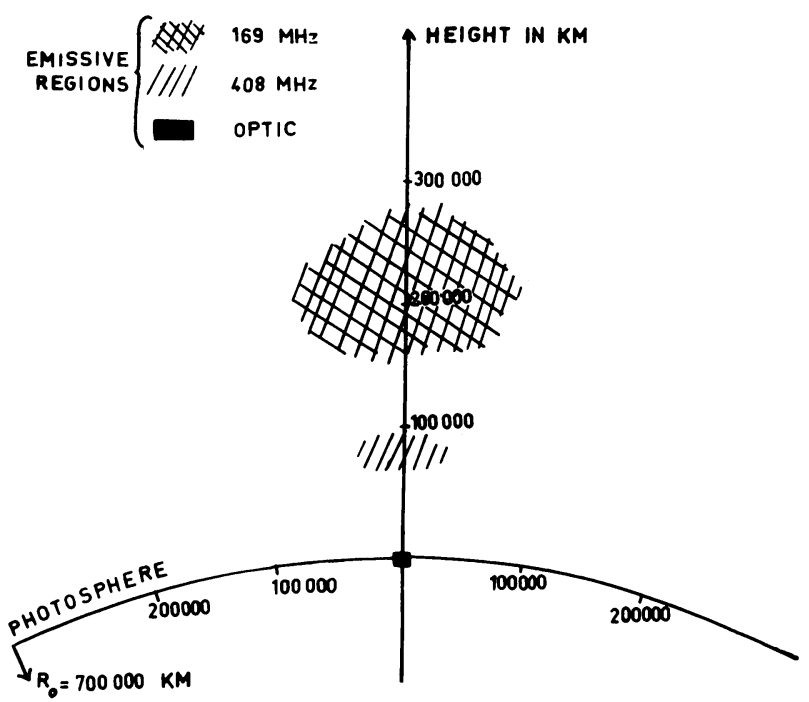

FIG. 6. Schematic structure above an active region (simple radio centre).

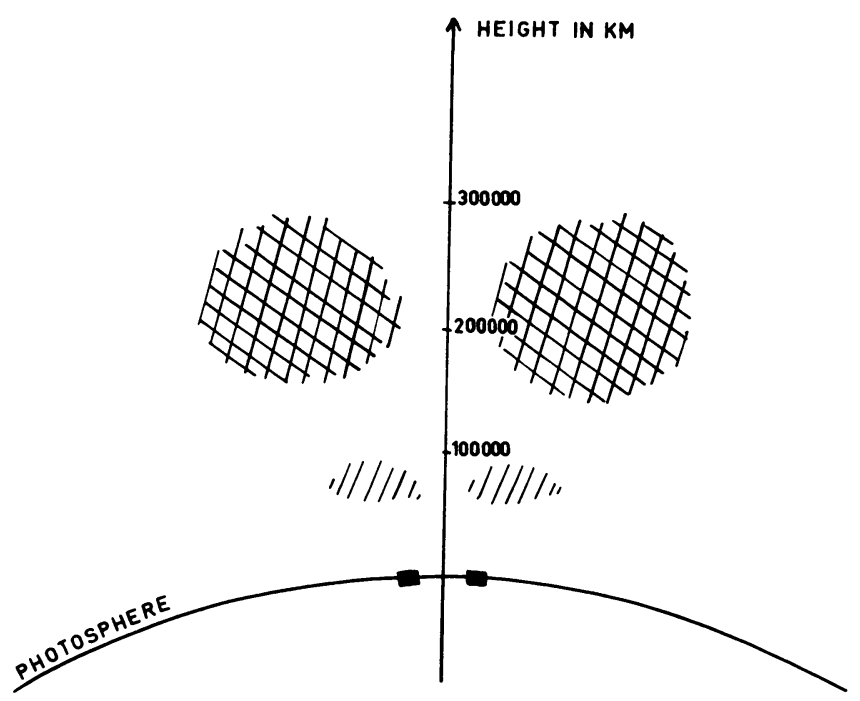

FIG. 7. Schematic structure for a double centre.

existence of an anomalous AR. Furthermore, one could put forward the theory that any radio centre is associated with a distinct eruptive zone.

Results obtained at 169 and $408 \mathrm{MHz}$ allow us to draw a schematic picture (Figures 6 and 7 ) of the structure of the active zones. The diameter increases with altitude (but 
nothing can be said about the extension in latitude). We cannot exclude the possibility that the simple centre might have a multiple structure not resolved by our instruments.

As to double centres, one would be tempted to identify them with a bipolar structure. However, since each of the individual centres seems associated with an eruptive region with its bipolar structure (as linked to an inversion line), one would rather think that the radio components of these centres are independent.

Another fact can confirm either one hypothesis or the other, that is the relation between the fluxes of the two radio centres. We have studied the comparative evolution of head and tail fluxes in the same double centres.

On November 8, 1965 and March 31, 1966, when the components were clearly visible for more than 1 hour, we have not found any correlation between fluxes of the two centres.

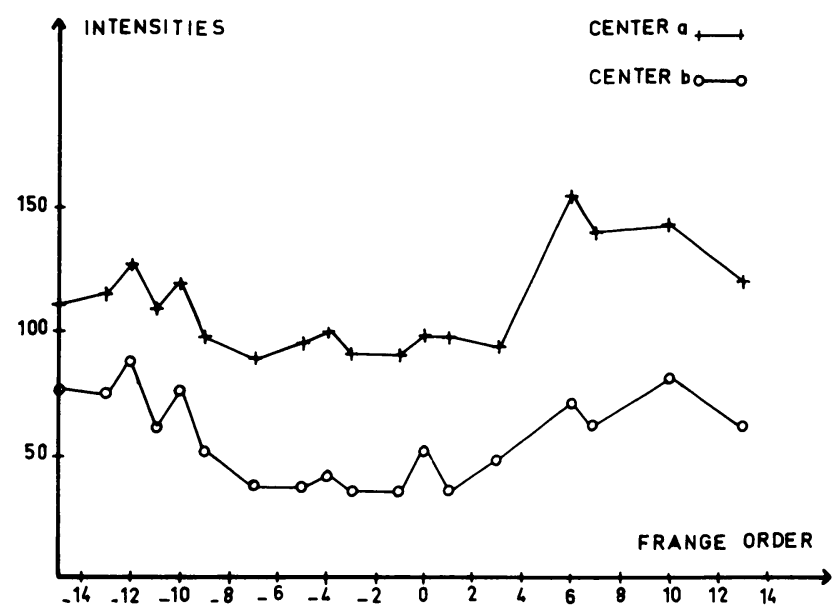

FIG. 8. Evolution of the components a and $b$ of the double centre on October 4, 1965.

Had this been confirmed later on, the second interpretation would be more valid.

Let us note, however, that on October 4, 1965 a strong correlation was found between the two fluxes (Figure 8), but it was a type-IV burst.

The study of the polarization of emissive centres at $408 \mathrm{MHz}$ with a better resolving power will permit us to add further details on the schematic structure described above.

\section{References}

Clavelier, B. (1967) Ann. Astrophys., 30, 895.

Le Squeren, A. M. (1963) Ann. Astrophys., 26, 97.

Martres, M. J., Michard, R., Soru-Iscovici, J. (1966) Ann. Astrophys., 29, 246. 


\section{DISCUSSION}

Koeckelenbergh: Je n'ai pas bien compris quelle était la résolution angulaire de votre interferomètre? Clavelier: $1: 7$ arc.

Koeckelenbergh: Est-ce un interferomètre lineaire?

Clavelier: Oui, 16 miroirs.

Elgaróy: When you observe a noise-storm source with double structure, is there any connection between changes in the leading and the preceding part of the source? (See the present paper) 